\title{
Studying the regulation of epicuticular wax biosynthesis in barley using isogenic WIN1/win1 lines generated by site-directed mutagenesis
}

\author{
Kolosovskaya E.V. ${ }^{1,2 *}$, Gerasimova S.V.1,2, Korotkova A.M. ${ }^{1}$, Hertig C. ${ }^{3}$, \\ Morozov S.V. ${ }^{4}$, Chernyak E.I. ${ }^{4}$, Domrachev D.V. ${ }^{4}$, Vikhorev A.V. ${ }^{1,2}$, Shmakov N.A. ${ }^{1}$, \\ Kochetov A.V. ${ }^{1,2}$, Kumlehn J. ${ }^{3}$, Khlestkina E.K. ${ }^{1,2,5}$ \\ ${ }^{1}$ Institute of Cytology and Genetics, SB RAS, Novosibirsk, Russia \\ ${ }^{2}$ Novosibirsk State University, Novosibirsk, Russia \\ ${ }^{3}$ Leibniz Institute of Plant Genetics and Crop Plant Research, Gatersleben, Germany \\ ${ }^{4}$ Novosibirsk Institute of Organic Chemistry, SB RAS, Novosibirsk, Russia \\ ${ }^{5}$ Vavilov Institute of Plant Genetic Resources, St. Petersburg, Russia \\ * email: kolosovskaya@bionet.nsc.ru
}

Isogenic lines with contrasting phenotypes are a perfect experimental model system for the elucidation of gene functions. The Cas9/gRNA-mediated knockout of the barley (Hordeum vulgare) WAX INDUCER 1 (HvWIN1) gene, that encodes an APETALA2/ETHYLENE-RESPONSIVE FACTOR, resulted in epicuticular wax deficiency and glossy sheath phenotype in primary mutants of cv. 'Golden Promise' and their progeny. In the M3 generation, six homozygous lines with two different mutations in the WIN1 gene were established. To assess the possible pleiotropic effects of its lossof-function, a comprehensive phenotyping of the WIN1/winl pair of isogenic lines was performed. As a result, developmental stages and major agricultural traits proved not affected by the mutation. The ultrastructure and biochemical composition of the affected wax were studied using scanning electron microscopy and gas chromatography coupled with mass spectrometry. The wax of leaf blades consists of products of an alcoholforming pathway and its amount is only slightly reduced in winl mutants. The main wax components of wild-type leaf sheaths are represented by diketones. By contrast, winl mutants accumulate a significantly lower total amount of wax on their leaf sheaths and exhibit a markedly altered wax composition; the abundance of diketones is significantly decreased, and the content of alcoholic substances is increased. A comparative transcriptome analysis of leaf blade and leaf sheath across control and mutant lines revealed strikingly different expression profiles of wax biosynthesis genes. Based on these data, it is concluded that the WIN1 transcription factor is a major and specific trigger of wax biosynthesis in leaf sheaths during generative development in barley.

Acknowledgements: The study is supported by the RSF (21-66-00012). 